International Journal of Engineering \&Technology, 7(3.12)(2018)711-714
International Journal of Engineering \& Technology
WPC
Website: www.sciencepubco.com/index.php/IJET
Research paper

\title{
Medical Image Watermarking Technique Using Image Interpolation in Transform Domain
}

\author{
S Priya ${ }^{1^{*}}$, B Santhi $^{2}$, J RajaMohan $^{3}$ \\ ${ }^{1,2}$ School of Computing, ${ }^{3}$ School of Electrical and Electronics Engineering, \\ SASTRA University, Tamil Nadu, India \\ *Corresponding Author E-mail: ${ }^{1}$ priya@ict.sastra.edu
}

\begin{abstract}
In telemedicine, medical data are shared across the world among different specialists for various purposes through an unsecured medium. So there is a need to protect the medical data during transmission. With the help of image watermarking techniques, medical images are protected along with the electronic patient information (EPI). This paper proposes a medical image watermarking, by applying wavelet transform, using an interpolation technique. EPI data is embedded within the transformed medical image to generate a watermarked image. At the extraction side, EPI data are extracted and medical image is reconstructed without any loss. The performance of the proposed method is analyzed using a peak signal to noise ratio (PSNR), mean absolute error (MAE) and structural similarity index (SSIM). The experimental result shows that the proposed method gives better results.
\end{abstract}

\section{Introduction}

Now a days, data security is playing a vital role in a multimedia world. Information is transmitted from one place to another place and during transmission, there is a chance to attack the information. So information security is needed. Two main types of data hiding methods are stegnography and watermarking. In both the techniques secret data is embedded within other multimedia data like audio, image, video, etc. stenography concentrate only on the secret data and watermarking concentrate on both secret data and cover data[1]. In telemedicine applications, medical images are transmitted from one location to another location for diagnosis purpose with patient data. On the receiver side, the original medical image is needed without any loss for correct diagnosis. Hence the watermarking technique is the most suitable for telemedicine applications to transmit the medical images [19]. In medical image watermarking, patient information is embedded within a medical image and transmitted to the receiver. At the receiver side, the patient information is extracted from the received medical image and original image extracted without any loss [2,3]. Several types of image watermarking techniques are present [1]. Medical image watermarking is performed on spatial and transform domains [4,5,14-16]. For copyright's purpose, Dharwadkar et al [6] implemented a reversible medical image watermarking technique with zero tolerance in a noisy channel. In order to provide confidentiality, integrity, and authentication (CIA) Eldayem [7] proposed a reversible watermarking technique using adaptive encryption standard (AES) algorithm and message digest-5 (MD5). The hash value is generated using MD5 and the watermark is encrypted using AES algorithm. The encrypted watermark and hash value are embedded within a medical image. At the extraction side hash value is verified then the data is extracted and medical image is received.
In the spatial domain, the image size is modified using interpolation techniques to embed a secret data within a scaled image for reversible data hiding without affecting the original pixel values. The secret data is embedded within a cover image using nearest mean interpolation method (NMI) developed by Jung and Yoo [8]. The cover image is scaled up using NMI and scaled image is divided into a number of nonoverlapping blocks. Then the secret data is embedded within each block. Lee and Hunag [9] proposed an efficient reversible data hiding technique is proposed using neighboring pixels maximum difference values to hide the secret data to improve the embedding capacity. Wang et al [10] proposed a reversible data hiding by dividing the image pixels into two groups. In one group secret data is embedded using an interpolation error and in another group secret data is embedded using histogram shifting in order to increase the embedding capacity.

Using shearlet transform a robust watermarking technique is proposed by Mardanpour and Chachooki [11]. First, the image is divided into a number of subbands using shearlet transform. Using the singular value decomposition (SVD) watermark is embedded within a subband. Chetan and Nirmala [12] implemented robust image watermarking using integer wavelet transform (IWT). The watermark is coded using block level coding and embedded within a wavelet sub band of the image. Medical image watermarking is developed by Mehto and Mehra [13] using discrete wavelet transform DWT and discrete cosine transform (DCT). First, the image is decomposed using DWT, then for high-frequency subbands, DCT is applied. The watermark is embedded in DCT coefficients. Priya et al [17] proposed medical image watermarking using two transforms.IWT decompose the image into different subband and gould transform recover the medical image without any loss. Lossless image recovery is achieved by using interpolation based data hiding technique[19]. This paper proposes a reversible watermarking technique using the image interpolation technique in the transform domain. 


\section{Proposed System}

In this section, an interpolation based medical image watermarking technique is proposed. The medical image size is reduced by half. Then the cover medical image is generated using integer wavelet transform (IWT). The size reduced medical image is decomposed into a number of subbands. Then each subband is scaled up to generate a cover image subband and patient information (watermark) is embedded within a cover image subband as shown in Figure-1.

$C(i, j)=I(i, j)$

$C(i, j+2)=I(i, j+1)$

$C(i+2, j)=I(i+1, j)$

$C(i+2, j+2)=I(i+1, j+1)$

The wavelet approximation subband of size $m X n$ is scaled up using any interpolation technique of size $2 \mathrm{mX} 2 \mathrm{n}$. The other subbands are resized to $2 \mathrm{mX} 2 \mathrm{n}$ by inserting one row and one column between two pixels. For example, consider highfrequency wavelet subband pixel at $(i, j)$ position, then the cover image is generated by inserting one pixel right to that pixel and one pixel down to that pixel also insert one pixel in a diagonal direction between two subband pixels using the following equations.

Let I be the high-frequency subband and $\mathrm{C}$ is the interpolated cover subband.

then the interpolated subband pixel value is calculated by using the following equations

$$
\begin{aligned}
& C(i, j+1)=|I(i, j)-I(i, j+1)|+I(i, j) \\
& C(i+1, j)=|I(i, j)-I(i+1, j)|+I(i, j) \\
& C(i+1, j+1)=(C(i, j+1)+C(i+1, j)+C(i+2, j+1)+C(i+1, j+2)) / 4
\end{aligned}
$$

After the interpolated pixels are identified for the subband, the difference (diff) between the interpolated pixel with the maximum value of the original pixels is calculated. Based on the difference (diff) value the length (l) of the embedded secret data is calculated. The watermark (wk) value is calculated from the corresponding decimal value of the binary secret data. Then it is added with the interpolated pixel. This embedding process is represented using equations 8-12.

$$
\begin{aligned}
& \max =(c(i, j), c(i, j+2), c(i+2, j), c(i+2, j+2)) \\
& \text { diff }=|\max -c(i, j)|
\end{aligned}
$$

$1=\log _{2}$ (diff)

$\mathrm{wk}=\operatorname{decimal}(1$ bit secret data)

$$
C^{\prime}=C+w k
$$

Finally, inverse integer wavelet transform is applied to generate a watermarked medical image (W). It is transmitted to the remote reconstructed without any loss. Algorithm 1 shows the proposed watermarking technique embedding and extraction procedure.
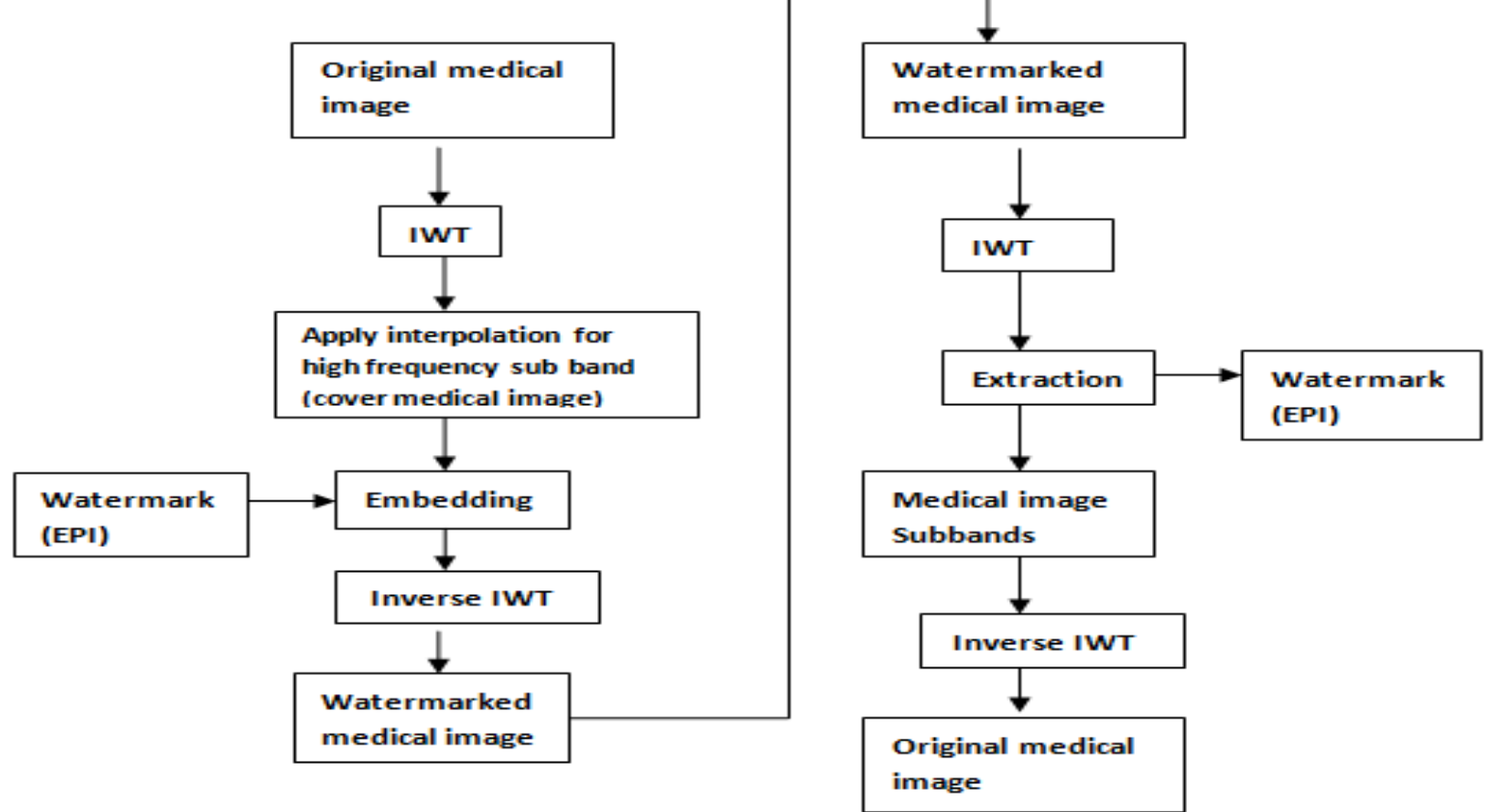

Fig. 1: Proposed watermarking technique 


\section{Algorithm 1:}

\section{Embedding Phase:}

1. Apply IWT to size reduced the original medical image.

2. For low frequency subband apply nearest neighbor interpolation to scale up the image.

3. For high frequency subband apply equations 1-7 to generate a cover image.

4. Embed watermarks (EPI) within an interpolated subband pixel.

5. Apply inverse IWT to generate a watermarked image.

\section{Extraction Phase}

1. Apply IWT to the watermarked image.

2. Repeat the embedding process to extracts the watermark (EPI).

3. Reduce the watermarked image to original medical image size.

4. Apply inverse IWT to reconstruct the original medical image.

\section{Experimental Results}

The proposed method performance is analyzed using various medical images with the size of 512 X 512 using MATLAB. Figure- 2 shows the original medical images. Using the proposed method interpolation, EPI is embedded within the wavelet subbands to generate a watermarked medical image. Figure-3 shows the watermarked images of Figure-2, visually there are no changes in the image quality, at the remote specialist side, watermark (EPI) is extracted and the original medical image is reconstructed.

$$
P S N R=10 \log _{10}\left(\frac{255^{2}}{M S E}\right),
$$

where MSE is the mean squared error,

$$
\begin{gathered}
M S E=\frac{\sum_{i=0}^{m-1} \sum_{j=0}^{n-1}\left(I(i, j)-\left(I^{\prime}(i, j)\right)\right)^{2}}{m n} . \\
M A E=\frac{1}{m n} \sum_{i=0}^{m-1} \sum_{j=0}^{n-1} I(i, j)-I^{\prime}(i, j), \\
S S I M=l(i, j) . c(i, j) . s(i, j),
\end{gathered}
$$

where 1 is the luminance, $\mathrm{c}$ is the chrominance and $\mathrm{s}$ is the structural component.

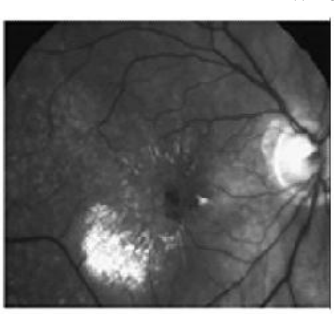

(a)

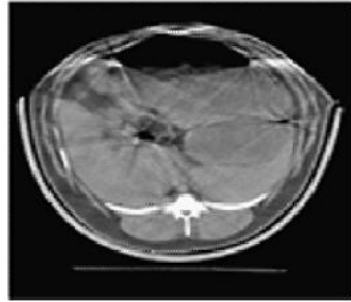

(b)

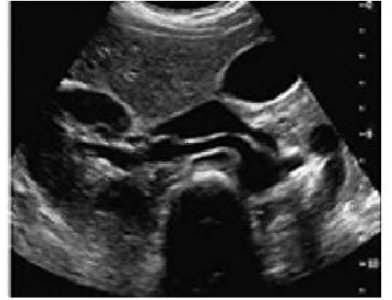

(c)

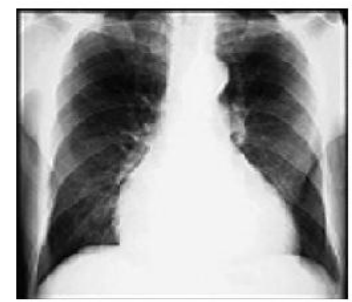

(d)

Fig. 2: Original medical images (a) Image1 (b) Image2 (c) Image3 (d) Image4

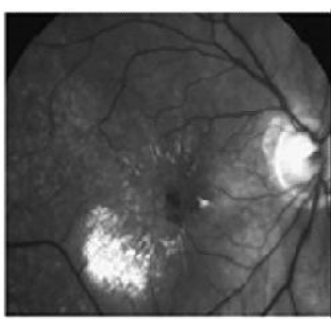

(a)

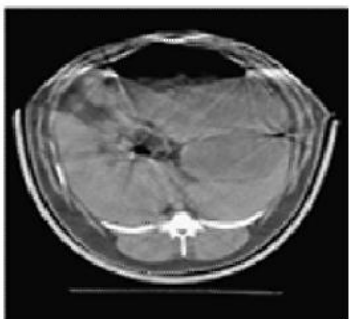

(b)

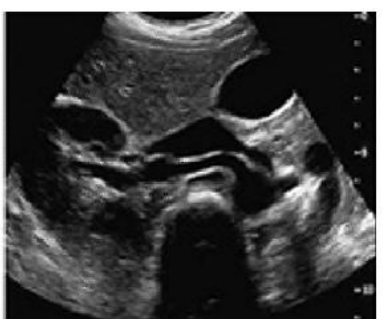

(c)

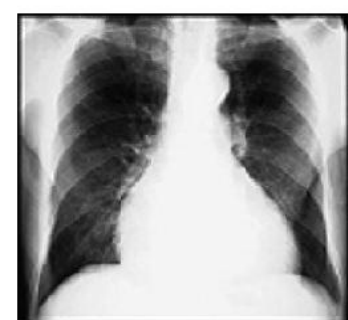

(d)

Fig. 3: Watermarked medical images (a) Image1 (b) Image2 (c) Image3 (d) Image4 
Table 1: Performance Measures of Proposed Technique

\begin{tabular}{|c|c|c|c|}
\hline Medical images & PSNR & MAE & 0.0546 \\
\hline Image1 & 48.67 & 0.0678 & \\
\hline Image2 & 47.45 & 0.9453 & 0.9346 \\
\hline Image3 & 48.32 & 0.065 & 0.9417 \\
\hline Image4 & 48.34 & 0.9541 & \\
\hline
\end{tabular}

Table 2: Comparison with the Existing Technique

\begin{tabular}{|c|c|c|c|c|c|c|}
\hline \multirow{2}{*}{ Medical Images } & \multicolumn{3}{|c|}{ Proposed method } & \multicolumn{3}{|c|}{$[8]$} \\
\hline & PSNR & MAE & SSIM & PSNR & MAE & SSIM \\
\hline Lena & 49.23 & 0.0837 & 0.9753 & 39.23 & 0.3213 & 0.8912 \\
\hline Baboon & 48.78 & 0.0863 & 0.9621 & 38.17 & 0.3842 & 0.8931 \\
\hline Airplane & 48.64 & 0.0854 & 0.9636 & 38.45 & 0.3848 & 0.8939 \\
\hline
\end{tabular}

The proposed method is compared with the existing interpolation technique [8] in table2 for different general images such as Lena, Baboon, Boat and Airplane. The PSNR and SSIM value for the proposed method is high and MAE value is low compare to existing techniques. It shows that the proposed method performs well compare to the existing techniques.

\section{Conclusion}

A medical image watermarking technique using image interpolation is proposed in this paper. By applying IWT to the original medical image, it is decomposed into four subbands. Using proposed interpolation technique subband size is changed and EPI is embedded. At the remote side, medical images and EPI data are extracted without any loss. PSNR value is high for the proposed method. SSIM value is nearly equal to 1 and MAE value less than 1. The experimental results show that the proposed method transmits the medical data in a secured manner.

\section{References}

[1] Priya S,Santhi B,Swaminathan P.Image Watermarking Techniques - A Review. Research Journal of Applied Sciences, Engineering and Technology.2012,4(14),pp. 2251-254.

[2] Giakoumaki A, Pavlopoulos S, Koutsouris D. Multiple image watermarking applied to health information management. IEEE Transactions on Information Technology in Biomedicine, 2006,10(4), pp722-732.

[3] Li X W, Kim S T. Optical 3D watermark based digital image watermarking for telemedicine. Optics and Lasers in Engineering.2013, 51(12),pp 1310-1320.

[4] Priya S,Santhi B,Swaminathan P.Study on Medical image watermarking Techniques. Journal of applied sciences.2013,14(14),pp. 1638-642.

[5] Kallel I F,Bouhlel M S,Lapayre J C. Improved Tian's Method for Medical Image Reversible Watermarking. GVIP Journal.2007,7(2),pp. 1-5

[6] Dharwadkar N V, Amberker B B, Panchannavar P B. Reversible Fragile Medical Image Watermarking with Zero Distortion. International Conference on computer and communication technology,2010,Allahabad.2010,pp. 248-54.

[7] 7.Abd Eldayem M M. A proposed security technique based on watermarking and encryption for digital imaging and communications in medicine. Egyptian Informatics Journal, 2013, 14(1),pp. 1-13.

[8] Jung K H, Yoo K Y. Data hiding method using image interpolation. Computer Standards \& Interfaces,2009,31(2), pp.465-470.

[9] Lee C F, Huang Y L. An efficient image interpolation increasing payload in reversible data hiding. Expert Systems with Applications 39(8) (2012) ,pp.6712-6719.

[10] Wang X T, Chang C C, Nauyen T S,Li M C. Reversible data hiding for high quality images exploiting interpolation and direction order mechanism. 2013, Digital Signal Processing 23 (2), pp. 569-577.

[11] Mardanpour M, Chachooki M A C. Robust transparent image watermarking with Shearlet transform and bidiagonal singular value decomposition AEU - International Journal of Electronics and Communications. 2015, 70(6), pp. 790-798.

[12] Chetan K R, Nirmala T. An efficient and secure robust watermarking scheme for document images using Integer wavelets and block coding of binary watermarks. 2015, Journal of Information Security and Applications, Volumes 24-25, pp13-24.

[13] Mehto A, Mehra N. Adaptive Lossless Medical Image Watermarking Algorithm Based on DCT \& DWT. Procedia Computer sciences2016, 78, International conference on Information Security \& Privacy. pp 89-94.

[14] Arsalan M, Malik S A, Khan S. Intelligent reversible watermarking in integer wavelet domain for medical images. 2012, journal of system and software, 85(4),pp 883-894.

[15] Das S, Kundu M K. Effective management of medical information through ROI-lossless fragile image watermarking technique. 2013 , computer methods and programs in biomedicine, 111(3), pp 662675.

[16] Fakhari P, Vahedi E, Lucas C. Protecting patient privacy from unauthorized release of medical images using a bio-inspired wavelet-based watermarking approach. 2011, 21(3), pp 433-446.

[17] Priya Selvam, Santhi Balachandran, Swaminathan Pitchai Iyer, Rajamohan Jayabal, Hybrid transform based reversible watermarking technique for medical images in telemedicine applications,2017,Optik 145,pp 655-671.

[18] Asaad F. Qasim, Farid Meziane a, Rob Aspin a, Digital watermarking: Applicability for developing trust in medical imaging workflows state of the art review,2018,Computer Science Review 27, pp45-60.

[19] Ki-Hyun Jung, A survey of interpolation-based reversible data hiding methods, 2017, Multimedia Tools and Applications,76,pp116. 